\title{
MICHAEL SELECTION THEOREM UNDER WEAK LOWER SEMICONTINUITY ASSUMPTION
}

\author{
KRZYSZTOF PRZESLAWSKI AND LONGIN E. RYBIŃSKI
}

(Communicated by Paul S. Muhly)

\begin{abstract}
We give a continuous selection theorem for convex-valued multifunctions satisfying slightly weaker lower semicontinuity assumptions than those which are adopted in the famous Michael Theorem [4] and in [1].
\end{abstract}

\section{INTRODUCTION}

Let $(Y \mid \cdot \|)$ be a normed linear space. Let $B=\{y \in Y \mid\|y\| \leq 1\}$ denote the closed unit ball and $B^{o}=\{y \in Y \mid\|y\|<1\}$ denote the open unit ball in $Y$. Denote $\mathscr{N}(Y)=\{C \subset Y \mid C \neq \varnothing\}, \mathscr{C}(Y)=\{C \in \mathscr{N}(Y) \mid C$ is convex $\}$, $\mathscr{C} \mathscr{C}(Y)=\{C \in \mathscr{C}(Y) \mid C$ is compiete $\}$. For $y \in Y, C \in \mathscr{N}(Y)$ set $y+C=$ $\{y+v \mid v \in C\}, r C=\{r v \mid v \in C\}$ for $r \in \mathbf{R}$.

Let $X$ be a topological space and $F: X \rightarrow \mathscr{N}(Y)$ be a multivalued mapping. A function $f: X \rightarrow Y$ such that $f(x) \in F(x) \quad\left(f(x) \in F(x)+\varepsilon B^{o}\right)$ for every $x \in X$ will be called a selection ( $\varepsilon$-approximate selection) of $F$.

A multivalued mapping $F: X \rightarrow \mathscr{N}(Y)$ is called lower semicontinuous (l.s.c.) at $x_{0} \in X$ iff for every $\varepsilon>0$ and $z \in F\left(x_{0}\right)$ there exists a neighborhood $U_{z}$ of $x_{0}$ such that

$$
z \in \bigcap\left\{F(x)+\varepsilon B^{o} \mid x \in U_{z}\right\} .
$$

$F$ is called Hausdorff lower semicontinuous (H.l.s.c.) at $x_{0} \in X$ iff for every $\varepsilon>0$ there exists a neighborhood $U$ of $x_{0}$ such that

$$
F\left(x_{0}\right) \subset \bigcap\left\{F(x)+\varepsilon B^{o} \mid x \in U\right\} .
$$

Following Deutsch and Kenderov [2], (see also [3]), we call $F$ almost lower semicontinuous (a.l.s.c.) at $x_{0} \in X$ iff for every $\varepsilon>0$ there exists a neighborhood $U$ of $x_{0}$ such that

$$
\bigcap\left\{F(x)+\varepsilon B^{o} \mid x \in U\right\} \neq \varnothing .
$$

Received by the editors December 29, 1988 and, in revised form, June 12, 1989.

1980 Mathematics Subject Classification. Primary 54C65; Secondary 54C60.

Key words and phrases. Lower semicontinuous multifunctions, continuous selections, convex sets. 
Following De Blasi and Myjak [1], we call $F$ weakly Hausdorff lower semicontinuous (w.H.l.s.c.) at $x_{0} \in X$ iff for every $\varepsilon>0$ and every neighborhood $V$ of $x_{0}$ there exist a neighborhood $U \subset V$ of $x_{0}$ and a point $x^{\prime} \in U$ such that

$$
F\left(x^{\prime}\right) \subset \bigcap\left\{F(x)+\varepsilon B^{o} \mid x \in U\right\} .
$$

$F$ is l.s.c. (H.l.s.c., a.l.s.c., w.H.l.s.c.) if $F$ is l.s.c. (resp. H.l.s.c., a.l.s.c., w.H.l.s.c.) at each point of $X$. Clearly if $F$ is H.l.s.c., then $F$ is both 1.s.c. and w.H.l.s.c., if $F$ is l.s.c., then $F$ is a.l.s.c. and if $F$ is w.H.l.s.c., then $F$ is a.l.s.c. In general, there are no other implications between these four concepts of lower semicontinuity (see examples below).

Assume now that $X$ is a paracompact space. The famous Michael Theorem [4], tells that if $F: X \rightarrow \mathscr{C} \mathscr{C}(Y)$ is l.s.c., then $F$ admits a continuous selection. The crux of the matter in Michael's proof is to show that $F$ has a continuous $\varepsilon$-approximate selection $f_{\varepsilon}$ for every $\varepsilon>0$ and then to show that the multivalued mapping $G: x \rightarrow F(x) \cap\left(f_{\varepsilon}(x)+\varepsilon B\right)$ possesses the same property. Deutsch and Kenderov [2, Theorem 2.4] proved that $F: X \rightarrow \mathscr{C}(Y)$ has a continuous $\varepsilon$-approximate selection iff $F$ is a.l.s.c. Unfortunately, for an a.l.s.c. $F$ and a continuous $\varepsilon$-approximate selection $f_{\varepsilon}$ of $F$ the multivalued mapping $G$ defined above may not be a.l.s.c. Involving an interesting by itself geometric lemma, De Blasi and Myjak [1] showed that if $F$ is w.H.l.s.c., then $G$ is also w.H.l.s.c. and therefore Michael's idea of the proof works for weakly Hausdorff lower semicontinuous multifunctions. Since lower semicontinuity does not imply weak Hausdorff lower semicontinuity (and vice versa), Michael's theorem is not a corollary to the De Blasi and Myjak result (and vice versa). However, careful reexamination of the De Blasi and Myjak paper allows us to state Michael's theorem under the assumption, (we call it the weak lower semicontinuity assumption), inspired by their concept of weak Hausdorff lower semicontinuity, but weaker than both lower semicontinuity and weak Hausdorff lower semicontinuity. Namely, we say that $F: X \rightarrow \mathscr{N}(Y)$ is weakly lower semicontinuous at $x_{0} \in X$, (w.l.s.c.), iff for every $\varepsilon>0$ and every neighborhood $V$ of $x_{0}$ there is a point $x^{\prime} \in V$ such that for every $z \in F\left(x^{\prime}\right)$ there is a neighborhood $U_{z}$ of $x_{0}$ such that

$$
z \in \bigcap\left\{F(x)+\varepsilon B^{o} \mid x \in U_{z}\right\}
$$

Straightforward checking shows that if $F$ is l.s.c., then $F$ is w.l.s.c. (set $x^{\prime}=$ $\left.x_{0}\right)$, if $F$ is w.H.l.s.c., then $F$ is w.l.s.c., and if $F$ is w.l.s.c., then $F$ is a.l.s.c. (fix arbitrary $V, x^{\prime} \in V$ and $z \in F\left(x^{\prime}\right)$ ). It is well-known that if $F$ is compact-valued, then $F$ is l.s.c. iff $F$ is H.l.s.c. The same proof can be used to show that if $F$ is compact-valued, then $F$ is w.l.s.c. iff $F$ satisfies the following w.H.l.s.c.-type condition (formally weaker than w.H.l.s.c.): for every $x_{0} \in X$, every neighborhood $V$ of $x_{0}$ and every $\varepsilon>0$ there exist a point $x^{\prime} \in V$ and a neighborhood $U$ of $x_{0}$ such that

$$
F\left(x^{\prime}\right) \subset \bigcap\left\{F(x)+\varepsilon B^{o} \mid x \in U\right\} .
$$


The following simple examples show that the other converses are not true in general.

Example 1. Let $X=\mathbf{R}, Y=\mathbf{R}^{2}, F(x)=\{(t, x t) \mid t \in(-\infty, \infty)\}$. Then $F$ is l.s.c., hence w.l.s.c., but not w.H.l.s.c. at each $x \in X$.

Example 2. Let $X=Y=\mathbf{R}$. Set $F(x)=\left[0, n^{-1}\right]$ for $x=q 2^{-n}$, with $q$ odd integer and $n$ a positive integer, $F(x)=[0,1]$ for the remaining $x \in \mathbf{R}$. Since for every $x_{0} \in \mathbf{R}$ there exists a sequence $\left(x_{k}\right)$ converging to $x_{0}, x_{k}=q_{k} 2^{-n_{k}}$, $n_{k} \rightarrow \infty$, it follows that $\bigcap\left\{F(x)+\varepsilon B^{o} \mid x \in\left(x_{0}-\delta, x_{0}+\delta\right)\right\}=\varepsilon B^{o}$ and $F$ is w.H.l.s.c., hence w.l.s.c., but not l.s.c. at every $x_{0} \in X$.

Example 3. Let $X=\mathbf{R}, Y=\mathbf{R}^{2}, F(x)=\{(t, 0) \mid t \in[0,+\infty)\}$ for $x=n^{-1}$, $n \in \mathbf{N}, F(x)=\{(t, x t) \mid t \in[-1,+\infty)\}$ for the remaining $x \in \mathbf{R} . F$ is neither w.H.l.s.c. nor l.s.c. at $x_{0}=0$, but $F$ is w.l.s.c. at $x_{0}=0$. To verify it, notice that

$$
\begin{aligned}
\left(-\varepsilon, \varepsilon(1+\delta) \delta^{-1}\right) \times\{0\} & \subset \bigcap\left\{F(x)+\varepsilon B^{o} \mid x \in(-\delta, \delta)\right\} \subset \\
& \subset\left(-\varepsilon, \varepsilon(1+\delta) \delta^{-1}\right) \times(-\varepsilon, \varepsilon)
\end{aligned}
$$

for every $\varepsilon>0, \delta>0$.

Example 4. Let $X=Y=\mathbf{R}$. Set $F(x)=[0,1]$ for $x$ rational and $F(x)=$ $[-1,0]$ for $x$ irrational. Then $F$ will be a.l.s.c., but not w.l.s.c. at every $x \in X$. Notice that $F$ has the continuous selection $f \equiv 0$.

Actually, by virtue of [2, Lemma 2.3, Theorem 2.7], if $X$ is paracompact, $Y=\mathbf{R}$ and $F: X \rightarrow \mathscr{C}(Y)$ has bounded closed values, then $F$ has a continuous selection iff $F$ is a.l.s.c. The next example shows that the same statement with $Y=\mathbf{R}^{2}$ is false.

Example 5. Let $X=\mathbf{R}, Y=\mathbf{R}^{2}, F(x)=\{(t, x t) \mid t \in[0,1]\}$ for $x$ irrational, $F(x)=\{(t, 0) \mid t \in[0,1]\}$ for rational $x \neq 0, F(0)=(1,0) . F$ is a.l.s.c., but $F$ has no continuous selection.

In $\S 2$ we give a repetition of the fundamental lemma of De Blasi and Myjak [1] in slightly modified and sharpened form and we state the continuous selection theorem.

\section{CONTINUOUS SELECTIONS OF WEAKLY LOWER SEMICONTINUOUS MULTIFUNCTIONS}

The basis for our considerations is constituted by certain stability properties of the intersections of convex sets with the balls. A comprehensive study on such properties will be published by the first author elsewhere. The proof of the lemma below, with $\gamma=2^{-1}$, has been given in fact in [1]. Although for the proof of our selection theorem, the statement for $\gamma=2^{-1}$ would suffice, the following general statement is more useful for other applications, (see $[6,7]$ ). 
Lemma 1. Let $(Y,\|\cdot\|)$ be a normed linear space and let $\gamma \in[0,1), \delta \in$ $[0,1-\gamma)$. If $E \cap(e+\gamma r B) \neq \varnothing$ for $r \geq 0, E \in \mathscr{C}(Y), e \in Y$, then

$$
(E+\delta r B) \cap(e+r B+\delta r B) \subset E \cap(e+r B)+M(\gamma, \delta) \delta r B,
$$

where $M(y, \delta)=1+2(1+\gamma)(1-\gamma-\delta)^{-1}$.

Proof. For $\delta=0$ or $r=0$ the statement is obvious, so suppose that $\delta>0$ and $r>0$. Without loss of generality we may assume that $e=0$ (since otherwise we can consider $E-e$ instead of $E)$. Fix an arbitrary $y \in(E+\delta r B) \cap(r B+\delta r B)$ and let $u \in E$ be such that $y-u \in \delta r B$.

Case 1. Suppose $\|y\| \leq(1-\delta) r$. Then $\|u\| \leq\|y-u\|+\|y\| \leq r$, hence $u \in E \cap r B$. Thus $y=u+(y-u) \in E \cap r B+\delta r B \subset E \cap r B+M(\gamma, \delta) \delta r B$.

Case 2. Suppose $(1-\delta) r<\|y\|$. Put $\lambda=(\|y\|-(1-\delta) r)(\|y\|-\gamma r)^{-1}$. Clearly $\lambda>0$ and

$$
\lambda<2 \delta(1-\gamma-\delta)^{-1},
$$

since $\gamma r<(1-\delta) r<\|y\|<(1+\delta) r$. Then put $u_{\lambda}=(1-\lambda) y$. We have $\left\|u_{\lambda}-y\right\| \leq \lambda\|y\| \leq \lambda(1+\delta) r$ and since $\lambda\|y\|-\lambda y r=\|y\|-(1-\delta) r$ we have also

$$
\left\|u_{\lambda}\right\|=(1-\lambda)\|y\|=(1-\delta-\lambda \gamma) r .
$$

Choose $z \in E \cap \gamma r B$ and put $v_{\lambda}=u_{\lambda}+\lambda z=(1-\lambda) y+\lambda z$. Then

$$
\left\|v_{\lambda}\right\| \leq(1-\delta-\lambda \gamma) r+\lambda \gamma r=(1-\delta) r
$$

and $\left\|v_{\lambda}-u_{\lambda}\right\| \leq \lambda \gamma r$. Take $w=(1-\lambda) u+\lambda z$. Clearly $w \in E$ and

$$
\left\|w-v_{\lambda}\right\|=(1-\lambda)\|y-u\| \leq(1-\lambda) \delta r .
$$

Therefore

$$
\|w\| \leq\left\|w-v_{\lambda}\right\|+\left\|v_{\lambda}\right\| \leq r(1-\delta+\delta-\lambda \delta)=r(1-\lambda \delta) \leq r .
$$

Thus $w \in E \cap r B$. Moreover,

$$
\begin{aligned}
\|w-y\| & \leq\left\|w-v_{\lambda}\right\|+\left\|v_{\lambda}-u_{\lambda}\right\|+\left\|u_{\lambda}-y\right\| \leq r((1-\lambda) \delta+\lambda \gamma+\lambda(1+\delta)) \\
& =r(\delta+\lambda(\gamma+1))<r \delta\left(1+2(1+\gamma)(1-\gamma-\delta)^{-1}\right) . \quad \text { Q.E.D. }
\end{aligned}
$$

Corollary 1. Let $L>1, d \geq 0, \sigma \geq 0$ and $\sigma<3^{-1}(L-1) d$ whenever $d>0$. For every $E \in \mathscr{C}(Y)$ and $e \in Y$ satisfying $E \cap(e+d B) \neq \varnothing$ there follows

$$
\left(E+\sigma B^{o}\right) \cap\left(e+L d B+\sigma B^{o}\right) \subset E \cap(e+L d B)+N(L) \sigma B^{o},
$$

where $N(L)=1+3(L+1)(L-1)^{-1}$.

Proof. If $d=0$ the statement follows trivially, so suppose $d>0$. If $E \cap(e+$ $d B) \neq \varnothing$, then setting $\gamma=L^{-1}, \delta=\sigma(L d)^{-1}, r=L d$ and applying Lemma 1 we obtain

$$
(E+\sigma B) \cap(e+L d B+\sigma B) \subset E \cap(e+L d B)+M\left(L^{-1}, \sigma(L d)^{-1}\right) \sigma B .
$$

Since $M\left(L^{-1}, \sigma(L d)^{-1}\right)=1+2(L+1)((L-1) d-\sigma)^{-1}<N(L)$, the statement follows now immediately. Q.E.D.

The next lemma gives the key of the proof of the selection theorem. 
Lemma 2. Assume that $L>1$. If $F: X \rightarrow \mathscr{C}(Y)$ is w.l.s.c. and $f: X \rightarrow Y$ is continuous, then for every continuous function $d: X \rightarrow[0, \infty)$ such that $F(x) \cap$ $(f(x)+d(x) B) \neq \varnothing$, the multivalued mapping $G: x \rightarrow F(x) \cap(f(x)+L d(x) B)$ is w.l.s.c.

Proof. Fix arbitrary $x_{0} \in X$, a neighborhood $V$ of $x_{0}$ and $\varepsilon>0$. Observe that if $d\left(x_{0}\right)=0$, then $G$ is 1.s.c., hence w.l.s.c. at $x_{0}$. Indeed, we have $G\left(x_{0}\right)=\left\{f\left(x_{0}\right)\right\}$; thus choosing a neighborhood $U$ of $x_{0}$ such that

$$
d(x)<2^{-1} \varepsilon \text { and } f\left(x_{0}\right) \in f(x)+2^{-1} \varepsilon B^{o} \quad \text { for } x \in U,
$$

we get

$$
f\left(x_{0}\right) \in \bigcap\left\{F(x) \cap(f(x)+L d(x) B)+\varepsilon B^{o} \mid x \in U\right\},
$$

since

$$
f(x) \in F(x) \cap(f(x)+d(x) B)+d(x) B \subset F(x) \cap(f(x)+L d(x) B)+2^{-1} \varepsilon B^{o}
$$

for every $x \in U$.

So let $x_{0} \in X$ be such that $d\left(x_{0}\right)>0$. Choose a $\sigma>0$ such that

$$
\sigma<\min \left\{6^{-1}(L-1) d\left(x_{0}\right), N(L)^{-1} \varepsilon\right\} .
$$

Choose a neighborhood $W$ of $x_{0}, W \subset V$, such that, for $x, x^{\prime} \in W$,

$$
\begin{aligned}
\left|d(x)-d\left(x^{\prime}\right)\right| & <\delta(2 L)^{-1}, \quad 2^{-1} d\left(x_{0}\right)<d(x), \\
\left\|f(x)-f\left(x^{\prime}\right)\right\| & <2^{-1} \sigma .
\end{aligned}
$$

Choose in $W$ a point $x^{\prime}$ such that for every $z \in F\left(x^{\prime}\right)$ there exists a neighborhood $U_{z}$ of $x_{0}$ such that

$$
z \in \bigcap\left\{F(x)+\sigma B^{o} \mid x \in U_{z}\right\} .
$$

Now take an arbitrary $w \in F\left(x^{\prime}\right) \cap\left(f\left(x^{\prime}\right)+L d\left(x^{\prime}\right) B\right)$. There exists a neighborhood $U_{w}$ of $x_{0}$ such that $w \in \bigcap\left\{F(x)+\sigma B^{o} \mid x \in U_{w}\right\}$. Set $W_{w}=W \cap U_{w}$. Since

$$
\begin{aligned}
\|w-f(x)\| & \leq\left\|w-f\left(x^{\prime}\right)\right\|+\left\|f\left(x^{\prime}\right)-f(x)\right\| \leq \\
& \leq L d\left(x^{\prime}\right)+2^{-1} \sigma<L d(x)+\sigma
\end{aligned}
$$

for $x \in W_{w}$, it follows that

$$
w \in \bigcap\left\{\left(F(x)+\sigma B^{o}\right) \cap\left(f(x)+L d(x) B+\sigma B^{o}\right) \mid x \in W_{w}\right\} .
$$

Clearly $\sigma<6^{-1}(L-1) d\left(x_{0}\right)<3^{-1}(L-1) d(x)$ for $x \in W_{w}$. Therefore, since $F(x) \cap(f(x)+d(x) B) \neq \varnothing$ for every $x$, then by Corollary 1 $\left(F(x)+\sigma B^{o}\right) \cap\left(f(x)+L d(x) B+\sigma B^{o}\right) \subset F(x) \cap(f(x)+L d(x) B)+N(L) \sigma B^{o}$, for every $x \in W_{w}$. Thus

$$
w \in \bigcap\left\{F(x) \cap(f(x)+L d(x) B)+\varepsilon B^{o} \mid x \in W_{w}\right\} \text {. Q.E.D. }
$$


Theorem 1. If $X$ is a paracompact topological space, $Y$ is a normed linear space and $F: X \rightarrow \mathscr{C} \mathscr{C}(Y)$ is w.l.s.c., then $F$ has a continuous selection.

Proof. Following Michael [4, Theorem 3.2"] (see also [1] or [2, Theorem 2.7]) we need only to construct a sequence of continuous mappings $f_{n}: X \rightarrow Y$ such that

$$
\begin{aligned}
& \left(1^{\circ}\right) f_{n}(x) \in F(x)+2^{-n} B^{o} \text { for } n=1,2, \ldots, x \in X, \\
& \left(2^{\circ}\right) f_{n}(x) \in f_{n-1}(x)+5 \cdot 2^{-n} B^{o} \text { for } n=2,3, \ldots, x \in X .
\end{aligned}
$$

Indeed, if a sequence $\left(f_{n}\right)$ for which $\left(1^{\circ}\right)$ and $\left(2^{\circ}\right)$ hold is defined, then $\left(f_{n}\right)$ is uniformly Cauchy, hence, by an easy appeal to $\left(1^{\circ}\right),\left(2^{\circ}\right)$ and the completeness of $F(x), x \in X$, uniformly convergent to a continuous mapping which is a continuous selection of $F$. Since $F$ is a.l.s.c., $F$ has a continuous $2^{-1}$. approximate selection $f_{1}$ by virtue of [2, Theorem 2.4]. Suppose we have defined $f_{1}, \ldots, f_{k}$ satisfying $\left(1^{\circ}\right)$ and $\left(2^{\circ}\right)$. Define $G: X \rightarrow \mathscr{C}(Y)$ by

$$
G(x)=F(x) \cap\left(f_{k}(x)+2^{-k+1} B\right) .
$$

By Lemma 2, (with $L=2, d(x) \equiv 2^{-k}, f(x)=f_{k}(x)$ ), $G$ is w.l.s.c., hence also a.l.s.c. Therefore $G$ has a continuous $2^{-k-1}$-approximate selection $f_{k+1}$. Then

and

$$
f_{k+1}(x) \in F(x)+2^{-k-1} B^{o}
$$

$$
\left\|f_{k+1}(x)-f_{k}(x)\right\|<2^{-k-1}+2^{-k+1} \text {. Q.E.D. }
$$

Lemma 3. Let $T, X$ be topological spaces. If $F: X \rightarrow \mathscr{N}(Y)$ is a w.l.s.c. multivalued mapping and $f: T \rightarrow X$ is a continuous and open mapping, then $F \circ f$ is w.l.s.c.

Proof. Fix $\varepsilon>0, t_{0} \in T$. Let $W$ be a neighborhood of $t_{0}$. Since $f(W)$ is a neighborhood of $f\left(t_{0}\right)$, there exists $x^{\prime} \in f(W)$ such that for every $z \in F\left(x^{\prime}\right)$ there exists a neighborhood $V_{z}$ of $f\left(t_{0}\right)$ such that $z \in \bigcap\left\{F(x)+\varepsilon B^{o} \mid x \in V_{z}\right\}$. Choose $t^{\prime} \in W$ such that $x^{\prime}=f\left(t^{\prime}\right)$, and then for every $z \in F\left(f\left(t^{\prime}\right)\right)$ set $U_{z}=f^{-1}\left(V_{z}\right)$. Then $z \in \bigcap\left\{F(f(t))+\varepsilon B^{o} \mid t \in U_{z}\right\}$. Q.E.D.

Corollary 2. Let $X$ be a topological space such that $X \times Y$ is paracompact. If $F: X \rightarrow \mathscr{C} \mathscr{C}(Y)$ is w.l.s.c. and $m: X \times Y \rightarrow \mathbf{R}$ is a continuous mapping such that $F(x) \cap(y+m(x, y) B) \neq \varnothing$, then for every $L>1$ there exists a continuous mapping $f_{L}: X \times Y \rightarrow Y$ such that $f_{L}(x, y) \in F(x)$ and $\left\|f_{L}(x, y)-y\right\| \leq$ $\operatorname{Lm}(x, y)$ for every $(x, y) \in X \times Y$.

Proof. Define a multivalued mapping $G_{L}: X \times Y \rightarrow Y$ by

$$
G_{L}(x, y)=F(x) \cap(y+\operatorname{Lm}(x, y) B) .
$$

We need only to show that $G_{L}$ has a continuous selection. The projections $\mathrm{pr}_{X}, \operatorname{pr}_{Y}$, defined by $\operatorname{pr}_{X}(x, y)=x, \operatorname{pr}_{Y}(x, y)=y$, are continuous and open. Hence, in particular, by virtue of Lemma $3, F \circ \mathrm{pr}_{X}$ is w.l.s.c. Since

$$
G_{L}(x, y)=\left(\left(F \circ \operatorname{pr}_{X}\right)(x, y)\right) \cap\left(\operatorname{pr}_{Y}(x, y)+L m(x, y) B\right),
$$


then by Lemma 2, $G_{L}$ is w.l.s.c. By Theorem $1, G_{L}$ has a continuous selection. Q.E.D.

Remark 1. In order to assure that $X \times Y$ is paracompact it suffices to assume, e.g., that $X$ is paracompact and perfectly normal (see [5]).

Remark 2 . One can readily verify (arguing by contradiction) that $F: X \rightarrow$ $\mathscr{N}(Y)$ is w.l.s.c. at $x_{0}$ iff for every neighborhood $V$ of $x_{0}$, every $\varepsilon>0$ and every (not necessarily continuous) selection $s$ of $F$ there exists a neighborhood $U$ of $x_{0}$ such that

$$
s(V) \cap \bigcap\left\{F(x)+\varepsilon B^{o} \mid x \in U\right\} \neq \varnothing .
$$

This observation suggests the following generalization. We say that $F$ is convex lower semicontinuous (c.l.s.c.) at $x_{0}$ if for every neighborhood $V$ of $x_{0}$, every $\varepsilon>0$ and every selection $s$ of $F$ there exists a neighborhood $U$ of $x_{0}$ such that

$$
\overline{\operatorname{co}} s(V) \cap \bigcap\left\{F(x)+\varepsilon B^{o} \mid x \in U\right\} \neq \varnothing .
$$

The proof of Lemma 2 may be repeated with slight changes, and consequently Theorem 1 remains valid, if $F$ is allowed to be convex lower semicontinuous instead of weak lower semicontinuous.

\section{REFERENCES}

1. F. S. De Blasi and J. Myjak, Continuous selections for weakly Hausdorff lower semicontinuous multifunctions, Proc. Amer. Math. Soc. 93 (1985), 369-372.

2. F. Deutsch and P. Kenderov, Continuous selections and approximate selections for set-valued mappings and applications to metric projections, SIAM J. Math. Anal. 14 (1983), 185-194.

3. F. Deutsch, V. Indumathi, and K. Schnatz, Lower semicontinuity, almost lower semicontinuity, and continuous selections for set-valued mappings, J. Approx. Theory 53 (1988), 266-294.

4. E. Michael, Continuous selections I, Ann. of Math. 63 (1956), 361-382.

5. _ـ A note on paracompact spaces, Proc. Amer. Math. Soc. 4 (1953), 831-838.

6. L. Rybinski, An application of continuous selection theorem to the study of the fixed points of multivalued mappings, J. Math. Anal. Appl. (to appear).

7. __ Weak lower semicontinuity and Carathéodory type selections, (to appear).

Department of Mathematics, Higher College of Engineering, Podgórna 50, 65-246 Zielona Góra, Poland 